enhance structuring and targeting of HIV testing services for youth.

Disclosure No significant relationships.

\section{P416 LONGITUDINAL RELATIONSHIP AND SEXUAL HEALTH OUTCOMES FOR ADOLESCENT AND YOUNG ADULTS WITH PELVIC INFLAMMATORY DISEASE}

\begin{abstract}
${ }^{1}$ Maria Trent*, ${ }^{2}$ Jamie Perin, ${ }^{2}$ Jacquelin Toppins, ${ }^{2}$ Julia Rowell, ${ }^{2}$ Steven Huettner,

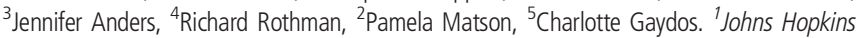
University School of Medicine, Ped Gen Pediatrics Adoles Medicine, Baltimore, USA; ${ }^{2} J o h n s$ Hopkins School of Medicine, Pediatrics, Baltimore, USA; ${ }^{3}$ Johns Hopkins School of Medicine, Pediatric Emergency Medicine, Baltimore, USA; ${ }^{4}$ Johns Hopkins School of Medicine, Adult Emergency Medicine, Baltimore, USA; ${ }^{5}$ Johns Hopkins School of Medicine, Baltimore, USA
\end{abstract}

10.1136/sextrans-2019-sti.502

Background Many adolescent and young women (AYA) remain in relationships with their sexual partners at 3-months postPID, but little is known about how those relationships continue to evolve and integrate risk reduction behaviors. The purpose of this study is to examine 12-month longitudinal sexual and reproductive health behaviors post-PID.

Methods Participants in the Technology Enhanced Community Health Nursing Study $(\mathrm{N}=286)$, a large randomized controlled trial of an intervention to improve PID outcomes were enrolled in quarterly post-trial telephone follow-up interviews. This analysis presents data from participants who completed 12 months of follow-up $(\mathrm{N}=72)$. Participants were queried about interim symptoms, sexual and reproductive behaviors, and clinical outcomes such as recurrent STI, pregnancy, chronic abdominal pain) and relationship status. Data were analyzed using descriptive and logistic regression analyses.

Results Of 72 participants who completed the 12-month post-trial follow-up, 33 were in the intervention group, and 39 were in the control group, and 42 (58\%) reported new partners in the last 12 months. Participants with new partners were not more likely to report having an STI than women without new partners (estimated odds ratio 1.6, 95\% CI $0.5,4.6, \mathrm{p}=0.470$ ) or to have used contraceptives in the past 12 months $(\mathrm{OR}=4.595 \%$ CI 0.3, 244.5, $\mathrm{p}=$ 0.301 ), but are more likely to have had lower abdominal pain (OR 5.6 95\% CI 1.8, 18.9, p=0.001). Most women $(86 \%)$ reported condom use in the prior 12 months, but there were no differences in condom use over the last year based on new partner status.

Conclusion Most AYA who experience PID acquire new partners during the year post-PID, but are not more likely to use condoms at 12 months. Given the increased association with lower abdominal pain, booster STI risk reduction strategies may be indicated to prevent associated sequelae among affected patients.

Disclosure No significant relationships.
PSO2 - POSTER VIEWING SESSION - TUESDAY

Tuesday, July 16, 2019

5:45 PM - 7:00 PM

\section{P417 HIGH INTEREST IN SYPHILIS PRE- AND POST-EXPOSURE PROPHYLAXIS AMONG GAY, BISEXUAL AND OTHER MSM IN VANCOUVER AND TORONTO}

${ }^{1}$ Laura Fusca*, ${ }^{2}$ Mark Hull, ${ }^{3}$ Patrick Ross, ${ }^{4}$ Troy Grennan, ${ }^{1}$ Ann Burchell, ${ }^{1}$ Ahmed Bayoumi, ${ }^{1}$ Darrell Tan. 'Li Ka Shing Knowledge Institute, St. Michael's Hospital, Centre for Urban Health Solutions, Toronto, Canada; ${ }^{2}$ University of British Columbia, Faculty of Medicine, Vancouver, Canada; ${ }^{3}$ University of British Columbia, Vancouver, Canada; ${ }^{4}$ British Columbia Center for Disease Control, Vancouver, Canada

10.1136/sextrans-2019-sti.503

Background Novel strategies for preventing bacterial sexually transmitted infections (STIs) are urgently needed. We assessed the acceptability of doxycycline-based syphilis preand post- exposure prophylaxis (PrEP/PEP) in a survey of gay, bisexual and other men who have sex with men (gbMSM) at risk.

Methods We recruited gbMSM from Toronto and Vancouver sexual health clinics during routine visits from 06/2018-08/ 2018. The questionnaire included demographics, sexual history/behaviours and knowledge/opinions regarding STI prevention. We analyzed data using descriptive statistics and constructed separate multivariable logistic regression models for willingness to use syphilis PrEP and PEP, with number of male partners in the last six months as the primary predictor, controlling for city, self-perceived syphilis risk, prior syphilis, concern about STI acquisition, number of different STIs previously diagnosed, depression, problem drug use and previous/ existing HIV PrEP/PEP use.

Results Among 424 participants, 56.4\%/43.6\% were recruited in Toronto/Vancouver. Median (interquartile range) age was $31.0(26.0,39.0)$ years, $61.7 \%$ had completed postsecondary education and $54.4 \%$ were White. Median number of male partners in the past 6 months was 6.0 (3.0, 13.0), and $18.2 \%$ had $\geq 1$ prior syphilis diagnosis. $60.1 \%$ and $44.1 \%$ indicated willingness to use syphilis PEP and PrEP respectively; $36.6 \%$ were unwilling to use either. Most participants were familiar with antibiotic resistance $(89.0 \%)$ and agreed that syphilis rates are rising in Canada (68.2\%), but only $55.4 \%$ believed they were at risk for syphilis. Agreement with the latter statement was significantly associated with willingness to use syphilis $\operatorname{PrEP} \quad(\mathrm{aOR}=1.6 ; 95 \% \mathrm{CI}=1.0$, 2.5), as was previous/existing HIV PrEP use $(\mathrm{aOR}=2.2 ; 95 \%$ $\mathrm{CI}=1.1,4.3)$ and being "very concerned" about STI acquisition $(\mathrm{aOR}=1.9 ; 95 \% \mathrm{CI}=1.0,3.4)$. Odds of being willing to use syphilis PEP were higher in Toronto versus Vancouver $(\mathrm{aOR}=2.0 ; 95 \% \mathrm{CI}=1.2,3.4)$ and increased with the number of different STIs previously diagnosed $\quad(\mathrm{aOR}=1.4 ; 95 \%$ $\mathrm{CI}=1.2,1.7)$.

Conclusion There is considerable interest in syphilis PrEP/PEP in gbMSM attending STI clinics in Toronto and Vancouver. Further research on such approaches is warranted.

Disclosure No significant relationships. 